\title{
Spatially Diverse ISAR Imaging for Classification Performance Enhancement
}

\author{
Stefan Brisken, Dietmar Matthes, Torsten Mathy, and Josef G. Worms
}

\begin{abstract}
One popular approach to the problem of NonCooperative Target Identification is the use of $2 D$ Inverse SAR images. Methods to successfully identify a target include the comparison of a set of scattering centers in the ISAR image to a database or the estimation of target dimensions. While working well in theory, these techniques face major difficulties in practice. In the conventional case of a monostatic radar, visibility of scattering centers varies with the target aspect angle due to fading. In this paper we examine that the visibility of scattering centers can be improved by incoherent addition of images from spatially distributed radars. The main focus lies in the description and results of a multistatic ISAR experiment carried out at Fraunhofer FHR. We display theoretically derived bistatic synchronization errors in a practical system and formulate additional multistatic synchronization requirements, necessary to add up the images.
\end{abstract}

Keywords-Inverse synthetic aperture radar, multistatic radar, non-cooperative target identification.

\section{INTRODUCTION}

$\mathbf{R}$ ECENT military conflicts have shown numerous examples of casualties due to friendly fire. In most cases, the reason for friendly fire is inadequate identification of vehicles or aircraft. Hence, there is a demand for a Non Cooperative Target Identification (NCTI) technique that can reliably distinguish between inimical and allied forces. This task, although addressed by many scientists in the past decade, has not been solved satisfactorily.

One approach to this problem is the comparison of scattering centers in $2 \mathrm{D}$ Inverse SAR images with a database, as demonstrated in [1]. But in the conventional case of a monostatic radar, this method is affected by the variation of visible scattering centers under different aspect angels. For this reason it is beneficial to integrate signals obtained from different aspect angles into a single image. In this paper we show a method of how to add an image obtained by a bistatic configuration to an image obtained by a monostatic configuration incoherently to improve the diversity of visible scattering centers. The technique was experimentally verified and can be used to add up images from additional transmitterreceiver pairs.

\section{EXPERIMENTAL SETUP}

The experiment comprises a software defined radar (SDR, transmitter and receiver) placed on a scissor lift, a target on

The authors are with the Fraunhofer Institute for High Frequency Physics and Radar Techniques FHR, Neuenahrer Strasse 20, 53343 Wachtberg, Germany (e-mails: \{stefan.brisken, dietmar.matthes, torsten.mathy, josef.worms $\} @$ fhr.fraunhofer.de). a turntable and a standoff wideband receiver. The scheme of the experimental setup can be seen in Fig.2.

The SDR is a very flexible system, in which the radar parameters like waveform, pulse repetition interval $P R I$ and bandwidth $B W$ can be adapted to the objective of the experiment. For the purpose of imaging, we choose to illuminate the target with chirped pulses of $B W=800 \mathrm{MHz}$ bandwidth with a center frequency at $f_{c}=8.9 \mathrm{GHz}$. Furthermore the same signal is transmitted to the bistatic receiver via a second antenna.

The bistatic receiver is an experimental ESM receiver based on a four channel digital oscilloscope. Both the scattered and the direct signals are downconverted with the same local oscillator. To keep the data volumes manageable we only record 5000 Samples on each new pulse.

Position accuracy is crucial in this experiment. Therefore we used a high precision GPS with Real Time Kinematics (RTK) option to measure positions of the SDR, the bistatic receiver and the turntable center. The position accuracy of the GPS device itself is $<2 \mathrm{~cm}$, but the inexact knowledge of transmit and receive antennas' phase centers lets us expand this error to $<10 \mathrm{~cm}$. The target rotates with an angular velocity of $1 \%$ s.

\section{SignAl PROCESSING}

The next step is to create High Range Resolution (HRR) profiles by matched filtering. For the SDR, this operation is performed online on FPGA while for the bistatic receiver the data has to be post-processed. The SDR furthermore applies a hamming window onto the HRR profiles.

The matched filter in the bistatic receiver can either be a local copy of the transmitted signal or the record of the directly transmitted signal. For the latter method sufficient signal to noise ratio is required and spurious signals as well as multipath effects have to be accounted for.

The relevant part of the HRR profiles is extracted and transformed back to the frequency domain.

Image reconstruction is then performed with the well known Back-Projection Algorithm. This algorithm is convenient to use because it contains no approximations and the same targetfixed coordinate system can be used for both images. Only trivial generalizations have to be made to apply it to the bistatic case. On the other hand it is computationally intensive and exact coordinates have to be given. The algorithm as applied in the general bistatic case is summarized here:

In the frequency domain, we expect from an isolated scatterer $S$ at $\left(x_{S}, y_{S}, z_{S}\right)$ a signal as

$$
s_{S}(\vartheta, f)=A(S) \cdot e^{-i \frac{2 \pi f}{c} \cdot\left(d_{1}+d_{2}\right)}
$$


where $A(S)$ is the amplitude of that scatterer and

$$
d_{1}=\left|\vec{R}_{1}-\left(\begin{array}{c}
x_{S} \\
y_{S} \\
z_{S}
\end{array}\right)\right|
$$

and

$$
d_{2}=\left|\left(\begin{array}{c}
x_{S} \\
y_{S} \\
z_{S}
\end{array}\right)-\vec{R}_{2}\right|
$$

is the distance from transmitter to the isolated scatterer and from the scatterer to the receiver respectively. The complete received signal is then expected to be the superposition of the signals from all scatterers:

$$
s_{\text {tot }}(\vartheta, f)=\sum_{x_{S}} \sum_{y_{S}} \sum_{z_{S}} s_{S}\left(\vartheta, f, x_{S}, y_{S}, z_{S}\right)
$$

For this reason, the complex conjugated phase of $s_{S}$ is taken as a test function in a matched filter approach to test the reflectivity of the point $(x, y, z)$. Finally, the reconstructed image results in

$\hat{A}(x, y(, z))=\sum_{\vartheta} \sum_{f} s(\vartheta, f) \cdot e^{i \frac{2 \pi f}{c}\left[d_{1}\left(\vec{R}_{1}, x, y, z\right)+d_{2}\left(\vec{R}_{2}, x, y, z\right)\right]}$

where $s(\vartheta, f)$ is now the experimentally received data set. This is valid for both the bistatic and the monostatic case, as the monostatic case is a special case of the bistatic case with $\vec{R}_{1}=\vec{R}_{2}$ and $d_{1}=d_{2}$

To remove sidelobes from the images we apply the CLEAN algorithm, first introduced in [2] in radio astronomy, nowadays a common tool in imaging radar. This algorithm assumes the target to be a set of point sources. It iteratively finds the point with highest amplitude in the image and computes the point spread function at this point. It subtracts the point spread function from the the image and adds a clean version (without sidelobes) of the point scatterer to a new map. After the desired number of iterations the residual map is added to the new map.

Let $\hat{A}_{1}$ be the image reconstructed from the SDR data and $\hat{A}_{2}$ that from the bistatic receiver. The amplitudes of the images are now scaled and the images can be added up incoherently to form a combined image, $\hat{A}_{t o t}$ :

$$
\hat{A}_{\text {tot }}=\frac{\left|\hat{A}_{1}\right|}{\left|\hat{A}_{1, \text { max }}\right|}+\frac{\left|\hat{A}_{2}\right|}{\left|\hat{A}_{2, \text { max }}\right|}
$$

An elaborate theoretical derivation of the resolution capabilities of bistatic ISAR can be found in [3]. Experimental results of these reconstructed images are presented in section V.

\section{SYNCHRONIZATION ISSUES}

One of the most difficult tasks in every multistatic system is the synchronization of transmitters and receivers. Accuracy requirements and impacts of synchronization errors have been calculated several times for bistatic radar, SAR and ISAR in the open literature, e.g. in [5]-[9]. [5] examines the impact of deterministic synchronization errors, while [6] and [8] focus on the limits and impacts of non-deterministic errors.

In section IV-A we briefly review these synchronization requirements and point out in what way they apply to a system

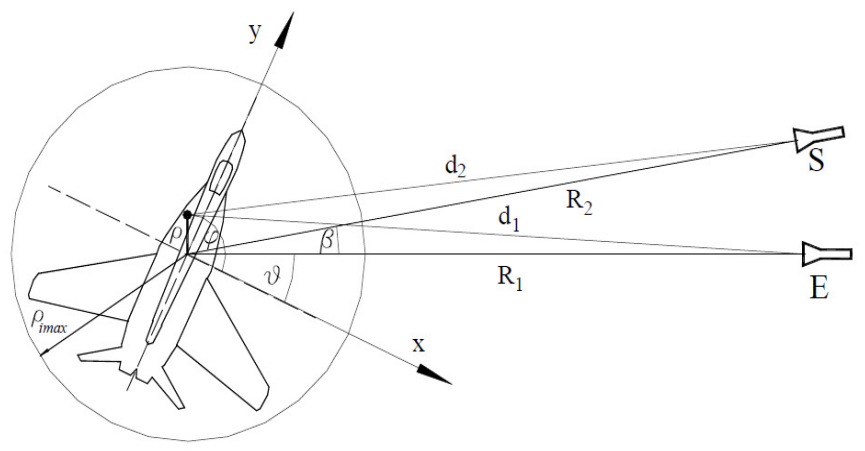

Fig. 1. Geometry of the turntable trial. The coordinate system $(x, y, z)$ is target fixed and the transmitter/receiver positions are given by $\vec{R}_{1,2}=$ $\left(\left|R_{1,2}\right| \cdot \cos \vartheta,\left|R_{1,2}\right| \cdot \sin \vartheta, z\right)$, where $\vartheta$ is the rotating angle. For the monostatic case is $\vec{R}_{1}=\vec{R}_{2}$ and therefore $d_{1}=d_{2} . \beta$ is called the bistatic angle. Image: Courtesy Thomas Vaupel [4], Fraunhofer FHR.

in practice, particularly the system used in the experiment described in this paper.

In addition to the bistatic synchronization requirements, we have to consider some multistatic synchronization requirements that are necessary for image fusion and might be tighter than the bistatic requirements. These additional requirements are evaluated in section IV-B.

\section{A. Bistatic Synchronization Issues}

Central to any aspect of synchronization is the phase deviation

$$
\Delta \phi=\phi_{r}-\phi_{t}
$$

It is important to understand what $\phi_{r}$ and $\phi_{t}$ are and where in the system deviations between them can occur. $\phi_{r}$ is the phase of the signal $S_{r}$ that is measured by the receiver ADC. On its way to the receiver ADC, $S_{r}$ is

1) synthesized by the transmitter DAC

2) upconverted with the transmitter LO

3) transmitted by the transmitter antenna

4) propagated through the atmosphere

5) reflected by the target

6) received by the receiver antenna

7) downconverted with the receiver LO

8) sampled by the receiver ADC

Taking all items listed above into account, a signal model $S_{t}$ is developed, that describes the expected signal at the receiver ADC. Therefore, $S_{t}$ is the signal that the matched filter and the SAR processor are fitted to. $\phi_{t}$ is the phase of $S_{t}$.

To keep phase deviations to a minimum, it is recommendable to phase lock transmitter DAC and LO to a transmitter master clock and receiver $\mathrm{LO}$ and $\mathrm{ADC}$ to a receiver master clock of high accuracy.

The first deviation from the modeled signal is then due to the imperfect nature of the Phase Locked Loop (PLL) that locks DAC, ADC and LOs to their master clock. This error is of a statistic nature (phase noise) and should be small compared to the total error if the PLLs are well designed. Typically, this phase noise is high frequency phase noise (meaning noise occurring at frequencies far away from the 
nominal oscillator frequency), while the low frequencies are rejected since disciplined by the master oscillator. See [10] for an elaborate discussion of PLL phase noise.

The most important influence on $\Delta \phi$ is usually caused by the phase deviation between the two master clocks $\Delta \phi_{m c}$. This deviation can be split up into a deterministic and a nondeterministic part

$$
\Delta \phi_{m c}=\Delta \phi_{m c, d}+\Delta \phi_{m c, n d}
$$

A quadratic phase drift $\Delta \phi_{m c, d} \propto T_{i n t}^{2}$ is caused if a linear frequency drift exists between the clocks and results in defocussed images. Phase deviation due to linear frequency change is therefore

$$
\Delta \phi_{Q}\left(T_{i n t}\right)=\frac{1}{2} \cdot 2 \pi \cdot K \cdot T_{i n t}^{2}
$$

with the frequency drift rate $K$ and integration time $T_{\text {int }}$. In [7] the maximum acceptable quadratic phase error is set to be $\pi / 2$. For a typical integration time of $T_{i n t}=10 \mathrm{~s}$, this is the case when

$$
K<\frac{1}{2 T_{\text {int }}^{2}}=5 \cdot 10^{-3} \frac{\mathrm{Hz}}{\mathrm{s}}
$$

or, as the common notation is,

$$
K / f_{c}<5 \cdot 10^{-8} \text { per day }
$$

with $f_{c}=8.9 \mathrm{GHz}$ being the RF center frequency in our experiment. This is easily feasible even for low cost OCXOs.

A quadratic phase error can also be induced by a mismatch between the chirp slopes of the actually received signal $\gamma_{r}$ and the chirp slope in the signal used for convolution in the matched filter process $\gamma_{t}$. We apply the same criterion as above and compute the maximum allowable chirp mismatch

$$
\begin{aligned}
\Delta \phi_{Q}\left(T_{P W}\right)=2 \pi\left(\left|\gamma_{r}-\gamma_{t}\right|\right) T_{P W}^{2} & <\frac{\pi}{2} \\
\Leftrightarrow\left|\gamma_{r}-\gamma_{t}\right| & <\frac{1}{4 T_{P W}^{2}}
\end{aligned}
$$

Inserting a pulsewidth of $T_{P W}=64 \mathrm{~ns}$ and the chirp slope

$$
\gamma_{r}=\frac{800 \mathrm{MHz}}{64 \mathrm{~ns}}
$$

we get

$$
\left|\gamma_{r}-\gamma_{t}\right|<6.1 \cdot 10^{13} \frac{\mathrm{Hz}}{\mathrm{s}}
$$

or

$$
\frac{\left|\gamma_{r}-\gamma_{t}\right|}{\gamma_{r}}<4.9 \cdot 10^{-3}
$$

Of course, chirp slope errors are not necessarily constant over $T_{P W}$, but in this paper we leave it at that and discuss on how to best fulfill this criterion for any kind of chirp slope error in section IV-C.

Non-deterministic phase errors $\Delta \phi_{m c, n d}$ cause so called statistical sidelobes that do not follow a deterministic pattern like common sidelobes. As a quality criterion, the integrated sidelobe ratio $I S L R$ can be computed as [6]:

$$
\int_{1 / T_{i n t}}^{\infty} 4 M^{2} L(f) d f
$$

where $L(f)$ is the single-sideband phase noise (1 Hz bandwidth) of the master clock and $\mathrm{M}$ is the ratio between $\mathrm{RF}$ frequency and master clock frequency. $L(f)$ is usually given in the master clock's specification. Apart from using ultra low phase noise oscillators, reducing $T_{\text {int }}$ is another effective mean (see section IV-C) to remain with an acceptable ISLR.

A phase error can also be induced by an imprecise measure of the antenna positions with respect to the rotation axis. The distance $d_{1}$ (see Fig.1) from the transmitter to a scatterer on the target at point $\vec{r}=(\rho \cos \phi, \rho \sin \phi, h)$ can be written as

$$
d_{1}=\sqrt{\left(\left|\overrightarrow{R_{1}}\right|+\rho \cos \psi\right)^{2}+(\rho \sin \psi)^{2}+h^{2}}
$$

with $\psi=\phi+\vartheta-\alpha$ and $\alpha$ as the angle between the transmitter's line of sight to the coordinate origin and the $\mathrm{x}$-axis and $h$ is the height of the transmitter above the scatterer in z-direction. An error in the measure of $\left|\vec{R}_{1}\right|$ of magnitude $\Delta R_{1}$ then results in an error for $d_{1}$ of

$$
\Delta d_{1}=\sqrt{\left(\left|\overrightarrow{R_{1}}\right|+\Delta R_{1}+\rho \cos \psi\right)^{2}+(\rho \sin \psi)^{2}+h^{2}}-d 1
$$

$\Delta d_{1}$ has a maximum for $\psi_{\max }=0^{\circ}$ and a minimum for $\psi_{\min }=90^{\circ}$ and $\psi_{\min }=270^{\circ}$. For values $\left|\overrightarrow{R_{1}}\right|=80 \mathrm{~m}$, $h=10 \mathrm{~m}, \Delta R_{1}=0.1 \mathrm{~m}$ and a maximum extend of the target of $\rho=4 \mathrm{~m}$, we get

$$
\Delta d_{1}\left(\psi_{\max }\right)=0.0995 \mathrm{~m}
$$

and

$$
\Delta d_{1}\left(\psi_{\min }\right)=0.0994 \mathrm{~m}
$$

We conclude that $\Delta R_{1}=0.1 \mathrm{~m}$ causes an almost constant range offset of about $\Delta d_{1}\left(\psi_{\text {min }}\right)=0.1 \mathrm{~m}$. The resulting phase error is then

$$
\Delta \phi=2 \pi \frac{2 \cdot \Delta d_{1}}{c}
$$

for the monostatic case and

$$
\Delta \phi=2 \pi \frac{\Delta d_{1}+\Delta d_{2}}{c}
$$

for the bistatic case. Of course, $\Delta d_{2}$ can be computed analog to $\Delta d_{1}$.

The deviation of $\Delta d_{1}$ is as small as $\Delta d_{1}\left(\psi_{\max }\right)-$ $\Delta d_{1}\left(\psi_{\text {min }}\right)=10^{-4} \mathrm{~m}$, even for a very large integration angle of $\Delta \vartheta=90^{\circ}$. This deviation is only $0.3 \%$ of a wavelength and can be neglected. The constant offset, however, will be important in section IV-B.

The influence of the height error $\Delta h$ can be computed in the same way. For $\Delta h=0.1 \mathrm{~m}$ we get $\Delta d_{1}\left(\psi_{\max }\right)=0,0096 \mathrm{~m}$ and $\Delta d_{1}\left(\psi_{\min }\right)=0.0104 \mathrm{~m}$.

An incorrect value for $\alpha$ rotates the monostatic image by $\Delta \alpha$. An incorrect value for $\beta$ rotates the bistatic image around $\Delta \beta / 2$.

Often neglected for ground based radars is the unexpected motion of antennas, e.g. due to wind. However, unexpected antenna motion over $T_{i n t}$ should not exceed $\lambda / 4$, with $\lambda$ being the transmitted wavelength. In our experiment, $\lambda / 4=0.75 \mathrm{~cm}$. 

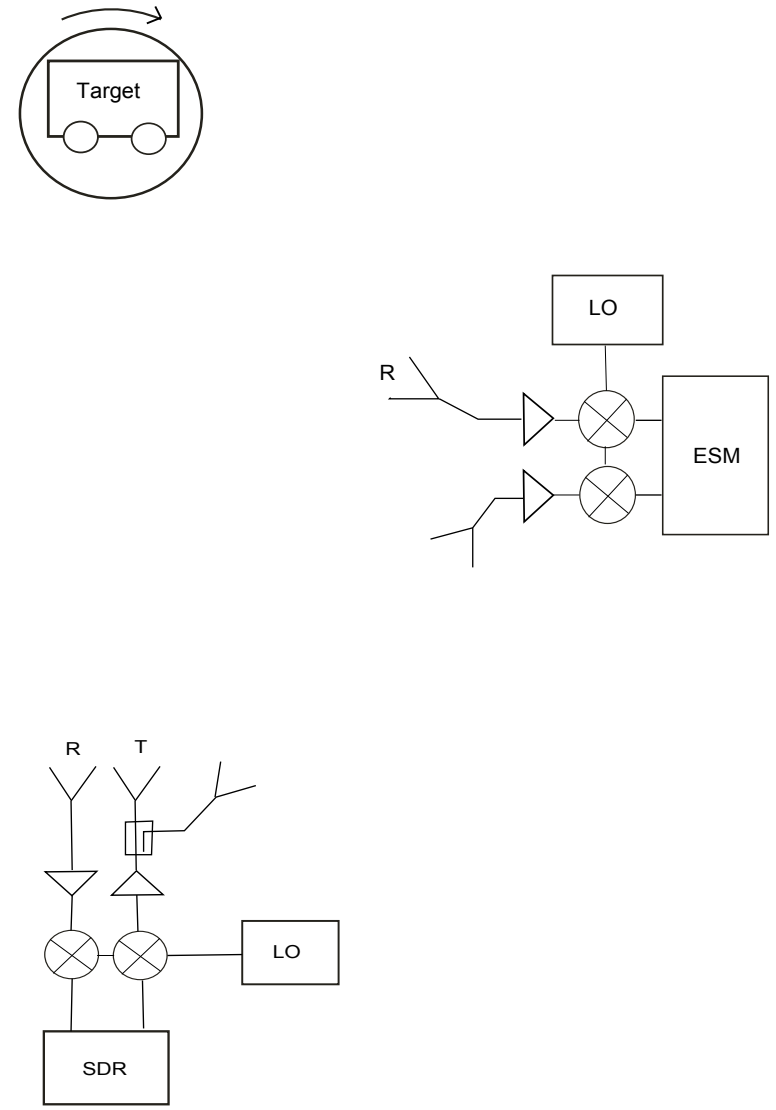

Fig. 2. Experimental scheme with the Software Defined Radar (bottom), the experimental ESM-Receiver (right) and the target on the turntable.

\section{B. Additional Multistatic Synchronization Issues}

With all bistatic synchronization requirements considered, we now look at the requirements necessary to fuse the images correctly. As a criterion for that, we define that a hypothetical point scatterer on the target must not be resolvable as two scatterers in the fused image. Therefore, the displacement in range and cross range direction $\Delta r$ between the images must be smaller than the images' range and cross range resolution, respectively

$$
\begin{aligned}
& \Delta r_{R}<\rho_{R} \\
& \Delta r_{C}<\rho_{C}
\end{aligned}
$$

We explicitly refer to a point scatterer because there may also be extended scatterers on the target, with their scattering center being in different positions in the monostatic and the bistatic image (Fig. 3). This is a desired effect. For the same reason we regard automatic image alignment with correlation methods as not useful and rather focus on achieving optimum synchronization.

In bistatic SAR, the range resolution is given by [11], [12]:

$$
\rho_{R}=\frac{c}{2 B W \cdot \cos (\beta / 2)}
$$

and the cross range resolution by

$$
\rho_{C}=\frac{c}{2 f_{0} \cdot \Delta \vartheta \cdot \cos (\beta / 2)}
$$

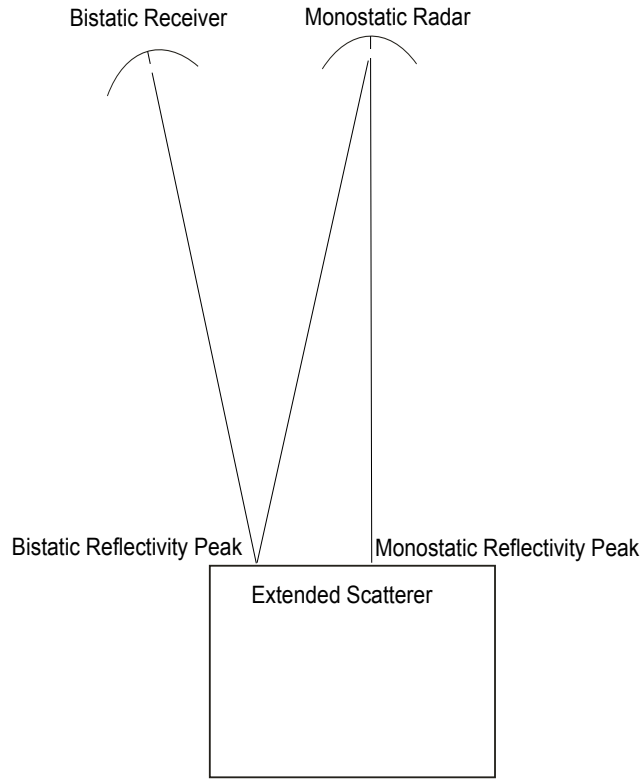

Fig. 3. Peak reflectivity point of an extended scatterer, monostatic and bistatic case. In the resulting ISAR image the scattering center appears at slightly different positions.

In our turntable geometry, resolution for both range and cross range is best for the monostatic case $\left(\beta=0^{\circ}\right)$. Computing the monostatic resolution with the values given above yields $\rho_{R}=$ $0.19 \mathrm{~m}$ and $\rho_{C}=0.1 \mathrm{~m}$ for a rotation angle of $\Delta \vartheta=10^{\circ}$. Note that bistatic range and cross range direction are rotated by $\beta / 2$ compared to monostatic. Hence, range and cross range errors of the same magnitude in both receivers do not cancel each other out completely.

Displacements can occur due to a constant frequency offset and incorrect transmitter/receiver positions. The effect of incorrect range and height measurements was already computed in section IV-A. While the displacement due to height error can be neglected in this case, the displacement due to an erroneous $\left|\vec{R}_{1}\right|$ value almost reaches the limit of the error margin.

An error in $\beta$ leads to an angular displacement between the two images of magnitude $\Delta \beta / 2$. The law of cosines provides the displacement of a scattering center at radius $\rho$ from the rotation axis as

$$
\Delta r_{S}=\sqrt{2 \rho^{2}\left(1-\cos \left(\frac{\Delta \beta}{2}\right)\right)}
$$

Note that this error is always in secantial direction across the circle with radius $\rho$, so it cannot generally be assigned to range or cross range. With a maximum target extent of $\rho_{\max }=4 \mathrm{~m}$, receiver distance from target $\left|\vec{R}_{2}\right|=80 \mathrm{~m}$ and a positioning error of $\Delta R_{S}=0.1 \mathrm{~m}$ in secantial direction, we get

$$
\Delta r_{S}=\frac{1}{2} \frac{\Delta R_{S} \cdot \rho_{\max }}{\left|R_{2}\right|}=2.5 \cdot 10^{-3} \mathrm{~m}
$$

which can also be neglected.

A range displacement can also be caused by a constant frequency offset $\Delta f$ of the two master clocks. This is in the very basic nature of radar. In our case, with $d=80 \mathrm{~m}$ and $\Delta d=0.19 \mathrm{~m}$, the maximum allowable relative frequency 
offset is then given by

$$
\frac{\Delta f}{f}=\frac{\Delta d}{d+\Delta d}=2.4 \cdot 10^{-3}
$$

Any given quartz watch would suffice for that

If not triggered on the direct signal, the two receivers pulse repetition intervals also depend on their individual master clock. For $P R I_{t} \neq P R I_{r}$ a virtual linear velocity in range direction $v_{D}$ superposes the rotational motion of the target:

$$
v_{D}=\frac{P R I_{t}-P R I_{r}}{P R I_{t}} \cdot c
$$

The range history of a scatter at position $(\rho, \phi)$ (compare Fig.1) would then be given by

$d_{1}\left(\rho, \phi, t_{n}\right)=\sqrt{\left(\left|\vec{R}_{1}\right|+\rho \cos \psi\right)^{2}+(\rho \sin \psi)^{2}+h^{2}}+v_{D} t_{n}$ with $\psi=\omega t_{n}+\phi+\alpha$ and $t_{n}=n \cdot P R I$ denoting the time of the $n$th pulse. If there is no compensation for $v_{D}$ in the image formation algorithm, the scatterer will appear displaced and defocussed in the resulting image. The displacement can be estimated by

$$
(\Delta \rho, \Delta \phi)=\operatorname{argmin}\left(\sum_{t_{n}}\left(d_{1}\left(\rho, \phi, t_{n}\right)-d\right)^{2}\right)
$$

with

$$
d=d_{1}\left(\rho+\Delta \rho, \phi+\Delta \phi, t_{n}\right)-v_{D} t_{n}
$$

This error, however, is well understood and can be compensated by methods of motion compensation (e.g. [7]) or by using the direct signal as described subsequently.

\section{Direct Signal vs. Local Copy}

Much of the criteria above can be relaxed when using the direct signal as a trigger. All inter-pulse effects fall apart and only the intra-pulse errors need to be considered. Coherency is not required over $T_{\text {int }}$ then, but only over the round trip time $\left(d_{1}+d_{2}\right) / c$. Low frequency phase noise on a scale $f<<$ $c /\left(d_{1}+d_{2}\right)$ does not contribute to the integrated sidelobe level any more.

However, the trigger process itself causes a jitter in the receiver PRI. By either finding a fixed scatterer in the HRR profiles or using the direct signal, a phase compensation can be applied to mitigate this jitter. If possible, it is beneficial to use the direct signal as a matched filter to generate the HRR profiles. Doing so cancels out any mismatch in chirp slopes, but special attention has to be paid to spurious signals and multipath effects.

\section{Results}

An example result for a bistatic angle of $\beta=25^{\circ}$ can be seen in Fig. 5.

Fig. 5a shows the image obtained by the bistatic receiver with a local copy of the transmitted signal used as matched filter. The image is dominated by sidelobes. These sidelobes are partially statistical sidelobes originating from phase jitter. These statistical sidelobes are removed when using the recorded direct signal as a matched filter in Fig. 5b. The

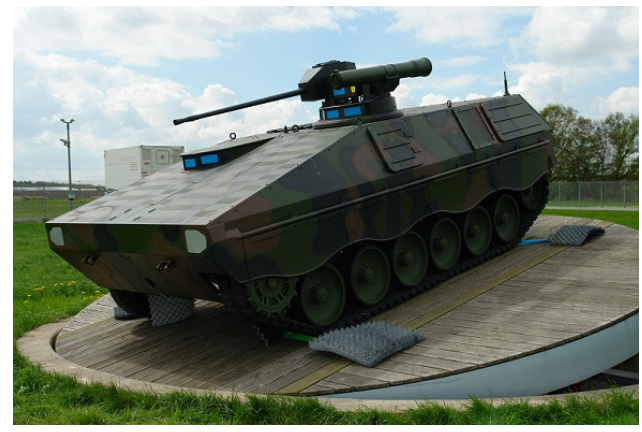

Fig. 4. Picture of the target: StanDCaM (Standard Decoy for Camouflage Materials), developed by WTD 52, Oberjettenberg, Germany. Surface material is metallized glass-fiber reinforced plastic.

residual sidelobes result from point spreading in the ISAR processing and can be removed with the CLEAN algorithm (Fig. 5c). In Fig. 5d we see the image obtained by the monostatic radar. Sidelobes are only visible in the cross range direction. This is because of the hamming window applied to the HRR profiles (section III). Fig. 5e shows the monostatic image after application of the CLEAN algorithm.

Due to the choice of a common coordinate system and oneto-one pulse correlation by triggering the receiver with the direct signal, the images from both receivers agree in position and orientation. This is the precondition to add up the images with Eqn. 1. The incoherent addition of the images $5 \mathrm{c}$ and $5 \mathrm{e}$ can be seen in Fig. $5 f$.

This example was chosen because it shows clearly additional scattering centers (at the top corner of the target) when adding up the images. The target shape looks more complete compared to the single images and clearly contains more information that can potentially be used for classification.

We furthermore see plenty of common scattering centers in both the monostatic and the bistatic images. A good example is the concentration of scatterers near the image center, which can be associated with the target's turret. With the approach chosen by us these common scatterers appear as the brightest in the combined image, while the brightness of scatterers only visible in one image falls behind. This effect, however, is small on the logarithmic $\mathrm{dB}$ scale.

Whether or not there actually is an improvement in the diversity of scattering centers varies from data set to data set and depends on aspect angle, bistatic angle, target properties and the value of the rotation angle $\Delta \vartheta$. This leads to certain application scenarios that are discussed in section VI.

\section{CONCLUSION}

It has been demonstrated that incoherent addition of ISAR images from spatially distributed receivers is feasible in an experiment without a common master clock and without cable connection. We identified the synchronization key issues in a practical, spatially distributed system and showed how to solve them.

For the example illustrated above, it has been shown that it is possible to improve the diversity of visible scattering centers. However, in a configuration where a monostatic radar is present, only a minority of the multistatic images show an 


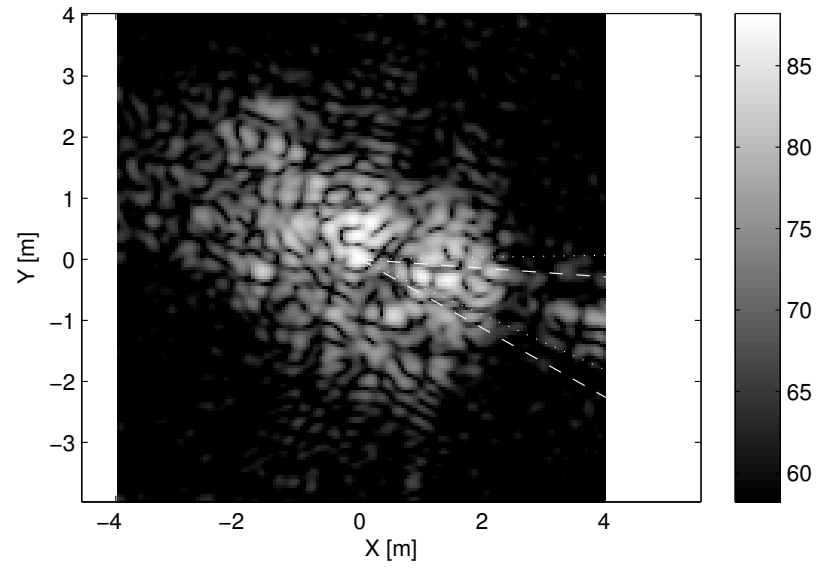

(a)

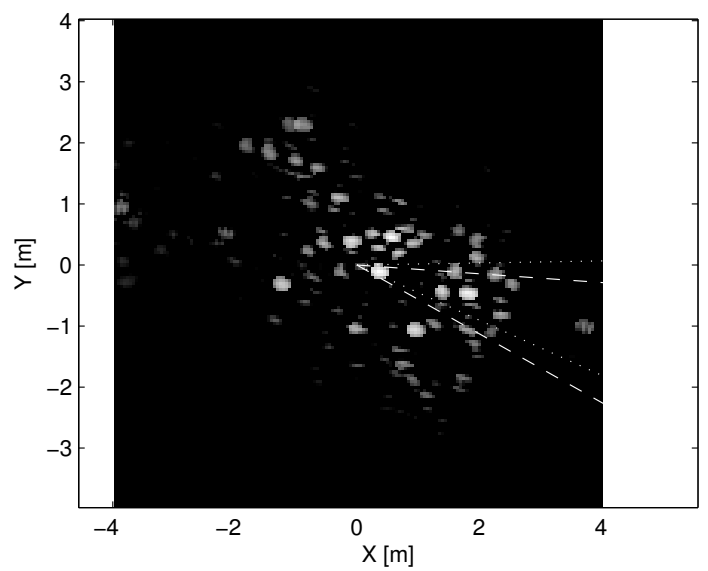

(c)

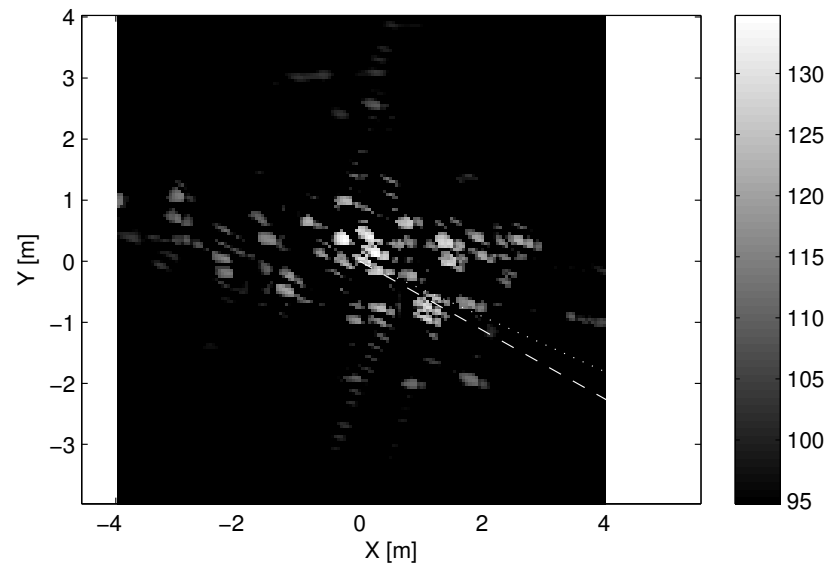

(e)

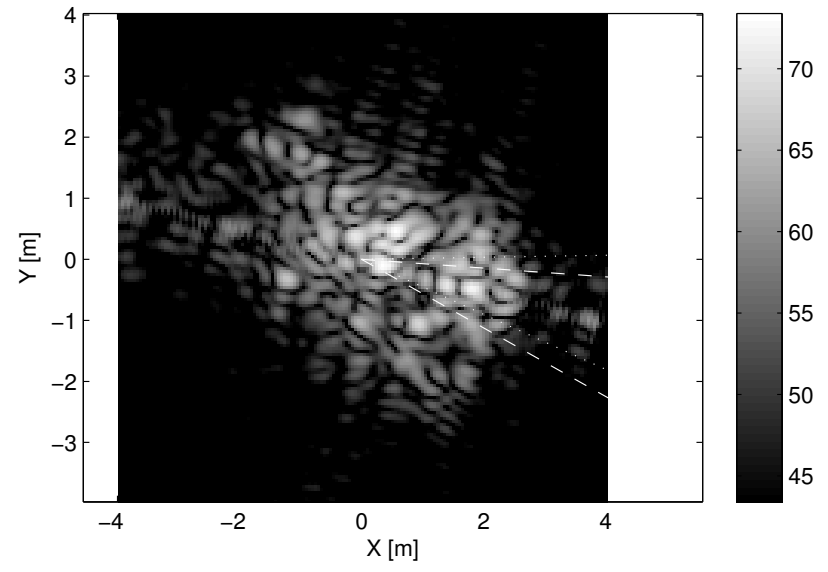

(b)

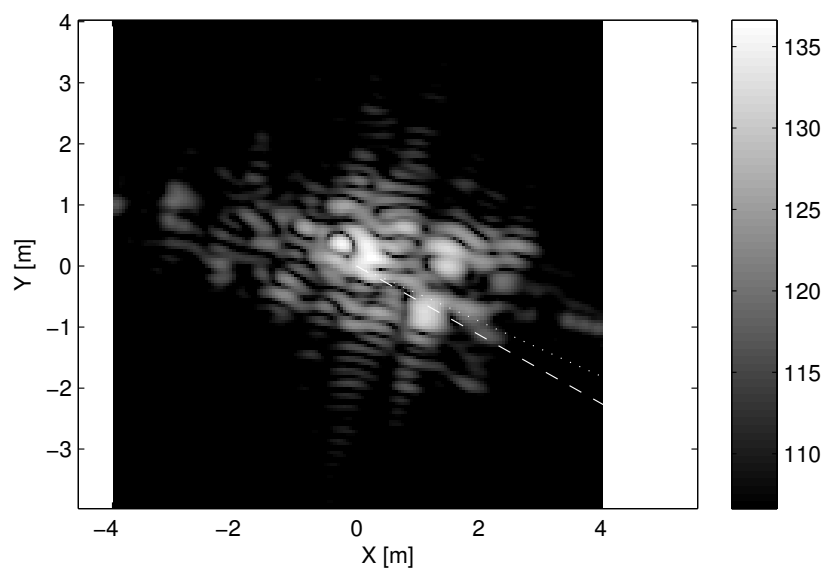

(d)

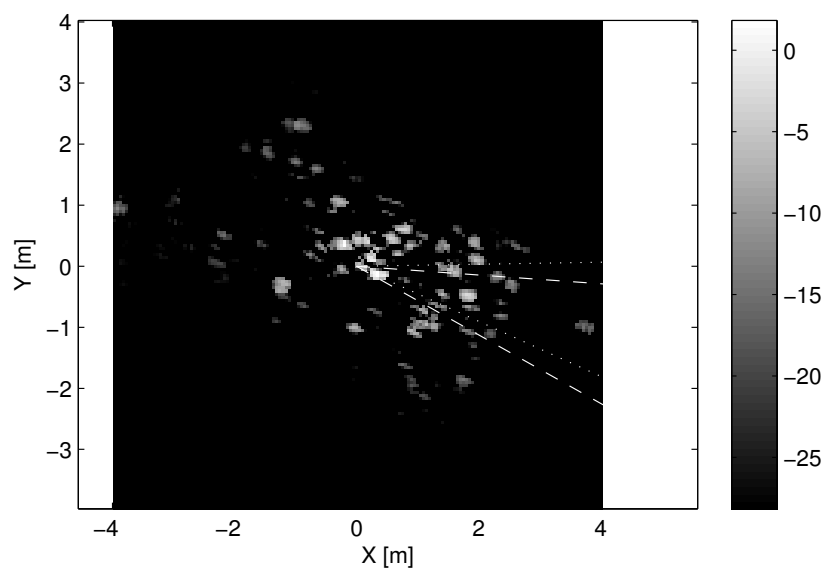

(f)

Fig. 5. Resulting ISAR images a) from the bistatic receiver with a local copy of the direct signal as matched filter, b) from the bistatic receiver with the recorded direct signal as matched filter, c) from the bistatic receiver after the CLEAN algorithm was applied, d) from the monostatic radar, e) from the monostatic radar after the CLEAN algorithm was applied, f) incoherent sum (Eqn. 1) of c) and e). The dashed and dotted lines show the start and stop position of the monostatic radar and the bistatic receiver in the target fixed coordinate system. The bistatic angle for this measurement is $\beta=25^{\circ}$, the rotation angle is $\Delta \vartheta=5^{\circ}$. Color scale in $\mathrm{dB}$ 
improvement over the monostatic image. This is, of course, due to the fact that a bistatic receiver cannot detect a scatterer that is not illuminated by the monostatic radar.

So this method is considered more suitable in scenarios where no monostatic image can be obtained and several bistatic receivers are used to create a multistatic image. These scenarios include unfortunate ISAR geometries, e.g. the target approaching the transmitter directly on the line of sight or scenarios in which the monostatic radar is jammed.

Another possible application for the presented method would be the imaging of stealth targets, as their design aims at minimizing the monostatic RCS, while the bistatic RCS can be compared to that of normal, unstealthy targets.

As the monostatic case is only a special case of the bistatic one, feasibility has also been shown for the scenarios named above. Adding up images from more than two receivers would then increase the desired effect and can be done with the same technique.

Adding more transmitters is also feasible with this technique, if the signals can be assigned to their originating transmitter.

Clearly the information content in the multistatic image is higher than in the monostatic one. Higher information content is generally considered to improve classification performance. If classification is improved in this particular case is yet to be shown.

\section{REFERENCES}

[1] K. Rosenbach and J. Schiller, "Identification of aircraft on the basis of 2-D radar images," in Radar Conference, 1995., Record of the IEEE 1995 International, May 1995, pp. 405-409.

[2] J. A. Högbom, "Aperture Synthesis with a Non-Regular Distribution of Interferometer Baselines," Astronomy and Astrophysics Supplement, vol. 15, pp. 417-+, Jun. 1974.

[3] N. Willis, Bistatic Radar, 2nd ed. Technology Service Corp., 1995.

[4] T. Vaupel and T. Eibert, "Comparison and application of near-field ISAR imaging techniques for far-field radar cross section determination," Antennas and Propagation, IEEE Transactions on, vol. 54, no. 1, pp. 144-151, Jan. 2006.

[5] J. W. Adams and T. M. Schifani, "Waveform error analysis for bistatic synthetic aperture radar systems," in Radar - 87; Proceedings of the International Conference, 1987, pp. 479-483.

[6] J. L. Auterman, "Phase stability requirements for bistatic SAR," in IEEE National Radar Conference, 1984, pp. 48 - 52.

[7] J. C. Kirk, Jr., "Bistatic SAR motion compensation," in International Radar Conference, 1985, pp. 360-365.

[8] G. Krieger and M. Younis, "Impact of oscillator noise in bistatic and multistatic SAR," Geoscience and Remote Sensing Letters, IEEE, vol. 3 , no. 3, pp. $424-428$, Jul. 2006.

[9] Z. Xiaoling, L. Hongbo, and W. Jianguo, "The analysis of time synchronization error in bistatic SAR system," in Geoscience and Remote Sensing Symposium, 2005. IGARSS '05. Proceedings. 2005 IEEE International, vol. 7, Jul. 2005, pp. 4619 - 4622

[10] D. Wolaver, Phase-Locked Loop Circuit Design. Prentice-Hall, 1991.

[11] C. Ozdemir, S. Demirci, B. Yilmaz, C. Ak, and E. Yigit, "A new and practical formulation of bistatic inverse synthetic aperture radar imaging and verification of the formulation using numerical examples," in Electrical and Electronics Engineering, 2009. ELECO 2009. International Conference on, Nov. 2009, pp. II-161 -II-164.

[12] Z. Zhu, Y. Zhang, and Z. Tang, "Bistatic inverse synthetic aperture radar imaging," in Radar Conference, 2005 IEEE International, May. 2005, pp. $354-358$ 